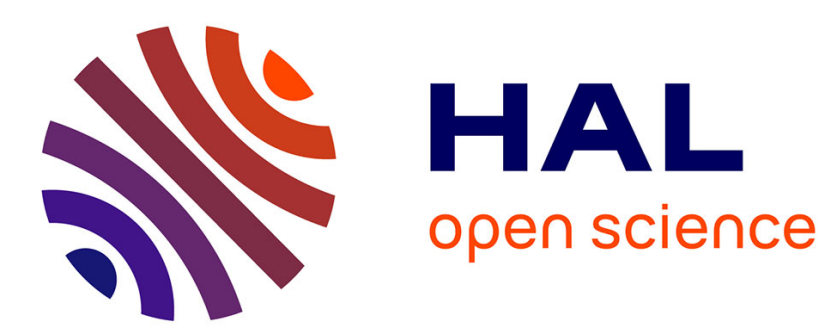

\title{
Jet dynamics post drop impact on a deep pool
}

Guy-Jean Michon, Christophe Josserand, Thomas Séon

\section{To cite this version:}

Guy-Jean Michon, Christophe Josserand, Thomas Séon. Jet dynamics post drop impact on a deep pool. Physical Review Fluids, 2017, 2 (2), pp.023601. 10.1103/PhysRevFluids.2.023601 . hal01501497

\section{HAL Id: hal-01501497 https: / hal.sorbonne-universite.fr/hal-01501497}

Submitted on 7 Apr 2017

HAL is a multi-disciplinary open access archive for the deposit and dissemination of scientific research documents, whether they are published or not. The documents may come from teaching and research institutions in France or abroad, or from public or private research centers.
L'archive ouverte pluridisciplinaire HAL, est destinée au dépôt et à la diffusion de documents scientifiques de niveau recherche, publiés ou non, émanant des établissements d'enseignement et de recherche français ou étrangers, des laboratoires publics ou privés. 


\title{
Jet dynamics post drop impact on a deep pool
}

\author{
Guy-Jean Michon, Christophe Josserand, and Thomas Séon \\ Sorbonne Universités, Université Pierre et Marie Curie and Centre National de la Recherche Scientifique, \\ UMR 7190, Institut Jean Le Rond d'Alembert, 4 Place Jussieu, F-75005 Paris France
}

(Received 13 June 2016; published 16 February 2017)

\begin{abstract}
We investigate experimentally the jet formed by the collapse of a cavity created by the impact of a drop on a pool of the same aqueous liquid. We show that jets can emerge with very different shapes and velocities, depending on the impact parameters, thus generating droplets with various initial sizes and velocities. After presenting the jet velocity and top drop radius variation as a function of the impact parameters, we discuss the influence of the liquid parameters on the jet velocity. This allows us to define two different regimes: the singular jet and the cavity jet regimes, where the mechanisms leading to the cavity retraction and subsequent jet dynamics are drastically different. In particular, we demonstrate that in the first regime, a singular capillary wave collapse sparks the whole jet dynamics, making the jet's fast, thin, liquid parameters dependent and barely reproducible. On the contrary, in the cavity jet regime, defined for higher impact Froude numbers, the jets are fat and slow. We show that jet velocity is simply proportional to the capillary velocity $\sqrt{\gamma / \rho_{l} D_{d}}$, where $\gamma$ is the liquid surface tension, $\rho_{l}$ the liquid density, and $D_{d}$ the impacting drop diameter, and it is in particular independent of viscosity, impact velocity, and gravity, even though the cavity is larger than the capillary length. Finally, we demonstrate that capillary wave collapse and cavity retraction are correlated in the singular regime and decorrelated in the cavity jet regime.
\end{abstract}

DOI: 10.1103/PhysRevFluids.2.023601

\section{INTRODUCTION}

From the first snapshots taken over a hundred years ago [1], followed by the celebrated high-speed photographs of Edgerton [2,3], and up to the world-famous commercial sequences on coffee drop impacts, the splash resulting from a drop impacting on a liquid surface is one of the most iconic images of fluid mechanics. This fame among the general public is undoubtedly due to its deeply attractive aesthetic, but drop impact also has a large field of applications. Inkjet printing [4], microfabrication of three-dimensional objects [5], underwater noise of rain [6], raindrop energy harvesters [7], forensic bloodstain pattern analysis [8], impact cratering [9], and the earthy smell, known as petrichor, present after a rain shower on a hot day [10] are a few among them. The diversity of these examples explains why drop impact has been studied by many authors $[11,12]$. Nevertheless, one aspect has been barely characterized so far: the jet dynamics and subsequent aerosols produced by a drop impact [13]. This question is crucial in different situations such as disease transmission [14] and liquid atomization [15] or its contribution to sea spray when a drop impacts the ocean $[11,16]$.

Indeed, when a drop impacts a liquid surface with a sufficiently low velocity it coalesces with the pool forming a vortex ring [17-20]. In this case, the free surface assumes its flat equilibrium position with no splash whatsoever, with a bouncing drop not being considered as splashing. As the drop impact velocity is increased, splashing regimes start [21]. The main features of splashing are the formation of a crown and the rise of a liquid column out of the middle of the crater. Indeed, the crater produced in the receiving liquid after impact is now deep enough, so that its retraction leads to a central jet springing up from its center [22]. This high speed jet can then fragment [23,24], sending one or more droplets upward [25,26]. At even higher impact velocity, the crown formed at impact can break up to produce a spray of numerous droplets [2,13,21]. At very high velocity, the crown rises as surface tension pulls it inward so as to form a canopy over the cavity. This canopy meets at 
the center and two opposite jets are ejected upward and downward. In this case, the downward jet stops the rise of the central jet generated from the cavity collapse [27,28]. Droplets are ejected in all these splashing regimes and they would all need to be quantified in order to improve the existing models of sea spray aerosols, for example. In the following, we focus on the characterization of the central jet dynamics.

To define the appropriate nondimensional parameters relevant in our study, we consider the normal impact of a spherical liquid drop on a flat liquid surface. The drop diameter is $D_{d}=2 R_{d}$, and the normal impact velocity is $V_{i}$. The liquid has a density $\rho_{l}$ and a dynamical viscosity $\mu_{l}$. The surface tension is denoted by $\gamma$ and the gravity by $g$. In the following, the drop impact dynamics is characterized by the Froude, Weber, and Reynolds numbers, defined as $\mathrm{Fr}=V_{i}^{2} / g D_{d}$, We $=\rho_{l} V_{i}^{2} D_{d} / \gamma$, and $\operatorname{Re}=\rho_{l} D_{d} V_{i} / \mu_{l}$, where inertia is balanced, respectively, by gravity, capillarity, and viscosity.

In this paper, the drop impact on a deep liquid pool is characterized under the prism of jet dynamics and droplet ejection. While jet dynamics and droplet ejection have been characterized for the collapse of cavities obtained by the bursting of bubbles [23,29,30] or large interface deformation [31,32], ours is a systematic study of cavities induced by drop impacts. In particular, in this configuration, the shape of the cavity is different, but above all, the cavity sizes are different, intermediate, typically between what has been studied so far, and we will see that this changes the subsequent jet dynamics.

Consequently, after a brief description of the experimental setup, the qualitative behavior of crater formation and subsequent jet formation will be discussed for different drop impact velocities. Then, the jet velocity and corresponding top drop size will be studied as a function of the drop impact velocity. The effect of liquid viscosity on jet dynamics will be quantified and the counter-intuitive behaviour of jet velocity with viscosity will be discussed. Furthermore, a surprising regime is identified at high impact velocity, where bubbles are entrapped and drops are ejected but, this time, without affecting the cavity retraction and jet dynamics. Finally, we show that although the cavity is gravity dominated for the high impact velocities investigated here, its retraction and the subsequent jet dynamics stay capillary driven.

\section{EXPERIMENTAL SETUP}

Our experiment consists of releasing a drop from a needle of internal diameter $0.84 \mathrm{~mm}$ located at heights ranging from $80 \mathrm{~mm}$ to $2.2 \mathrm{~m}$ above a deep pool of the same liquid and recording the collapsing cavity and the subsequent upward jet. Figure 1 presents a schematic view of the experiment and summarizes the main physical parameters.

Drops are quasi-steadily formed using a syringe. The drop diameter is taken such that the Froude and Weber numbers are equal for water drops. This imposes the drop diameter to be equal to the so-called capillary length: $D_{d}=\sqrt{\gamma / \rho_{l} g}$. In water, with a surface tension $\gamma=72 \mathrm{mN} \mathrm{m}^{-1}$ and a density $\rho_{l}=1000 \mathrm{~kg} \mathrm{~m}^{-3}$, our drop diameter is $D_{d}=2.7 \mathrm{~mm}$. Note that, in this case, the terminal velocity $\left(V_{i}=8.9 \mathrm{~m} \mathrm{~s}^{-1}\right)$ would be reached after a free fall of around $15 \mathrm{~m}$. At this height the drop impact does not produce a unique jet but rather a complicated splash shaping a canopy $[27,28]$, which is not considered in this paper. The liquid is contained in a transparent tank taken sufficiently large $\left(85 \times 105 \mathrm{~mm}^{2}\right.$ and $45 \mathrm{~mm}$ deep $)$ to rule out the confinement effects. The liquids used in this study include ten water-glycerol mixtures of viscosity in the range $\mu_{l}=1-20 \mathrm{mPa}$ s, surface tension $\gamma=64-72 \mathrm{mN} \mathrm{m}^{-1}$, and density $\rho_{l}=1000-1180 \mathrm{~kg} \mathrm{~m}^{-3}$ and ethanol in the last section $\left(\mu_{l}=1.2 \mathrm{mPas}, \gamma=22 \mathrm{mN} \mathrm{m}^{-1}\right.$, and $\left.\rho_{l}=780 \mathrm{~kg} \mathrm{~m}^{-3}\right)$. The cavity collapse and jet dynamics are analyzed through close-up high-speed imagery. Macro lenses and extension rings allow us to record with a definition reaching $20 \mu \mathrm{m}$ per pixel. Images are obtained at 8000 frames per second using a digital high-speed camera (Photron SA-5). The ejection speed $V_{j}$ is measured when the tip of the jet or the drop passes the mean water level. All the experiments are carried out at room temperature of $19 \pm 0.5^{\circ} \mathrm{C}$. 


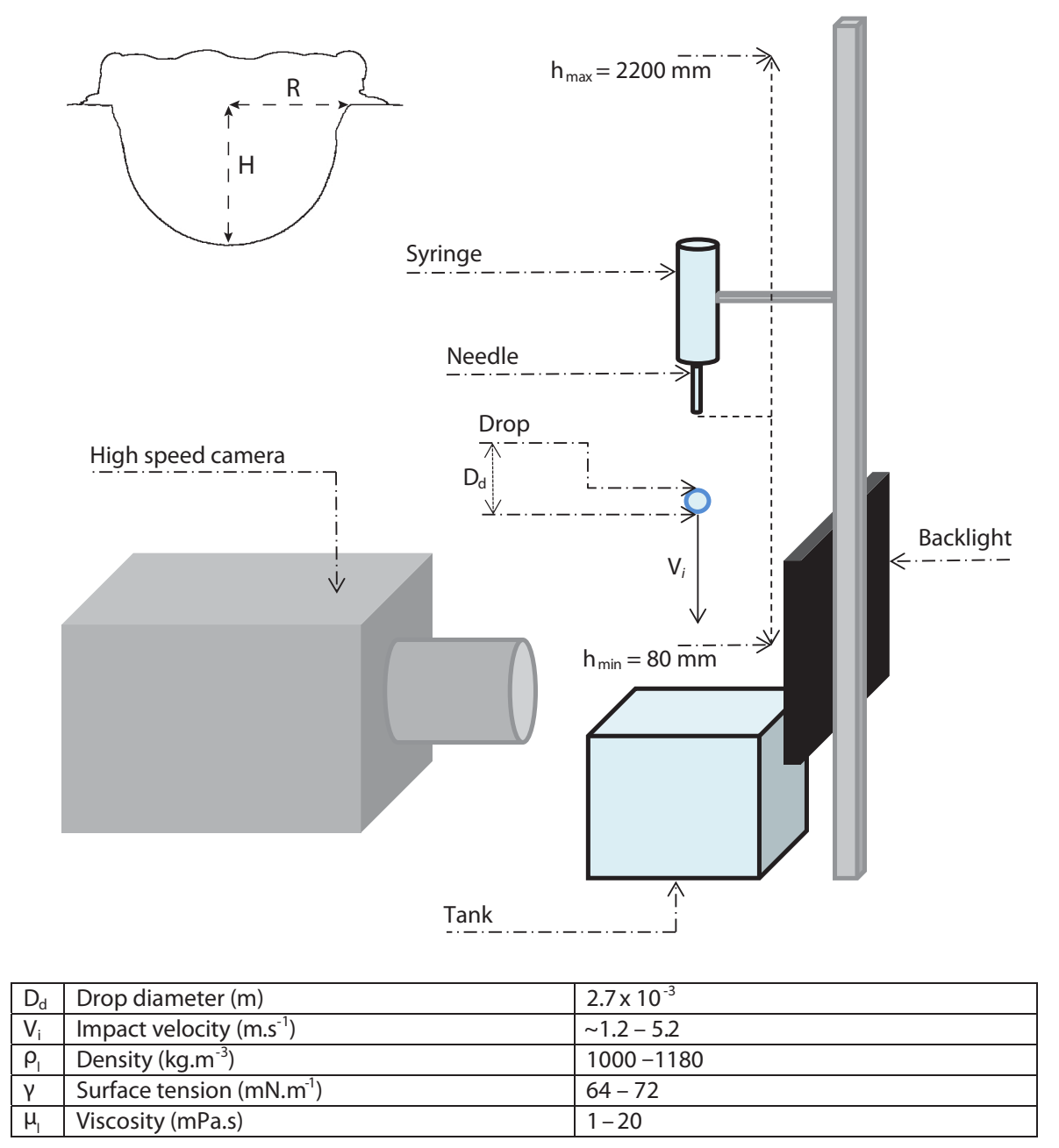

FIG. 1. Schematic view of the experiment and the values of the main physical parameters used. In the top left corner is a schematic of the cavity, defining the depth $H$ and radius $R$ of the cavity.

\section{QUALITATIVE STUDY}

When an opened cavity is submillimeter size, the restoring force which tends to bring this hole back to a flat equilibrium is capillary driven [29]. On the other hand, when a cavity is larger than a few centimeters, the relaxation process is a priori gravity driven since capillarity should become irrelevant [31,32]. In our drop impact experiments, typical cavity dimensions (heights $H$ and widths $2 R$ ) range from 5 to $15 \mathrm{~mm}$, so that both gravity and capillarity might play a role in the relaxation process. An insight into the respective role of each effect can be gained using a simple energy balance. First, noting that the dynamics of crater formation can be considered as inviscid [33,34], we disregard viscous effects in the following. The drop kinetic energy right before impact, $T=\frac{1}{2} M_{d} V_{i}^{2}$ (with $M_{d}=4 / 3 \pi \rho_{l} R_{d}^{3}$ ), is mainly converted, for the cavity shaping, into both gravitational potential energy $U \sim \frac{\pi}{2} \rho_{l} g H^{2} R^{2}$ and capillary energy $C \sim 2 \pi \gamma H R$, where $H$ and $R$ are respectively the maximum depth and radius of the cavity, as described in the top left corner of Fig. 1 . Note that the cavity-shape-dependent prefactor has been taken equal to the one of a cylinder for the sake of simplicity. In particular, it is known that the geometry of the cavity can be influenced by the prolate 


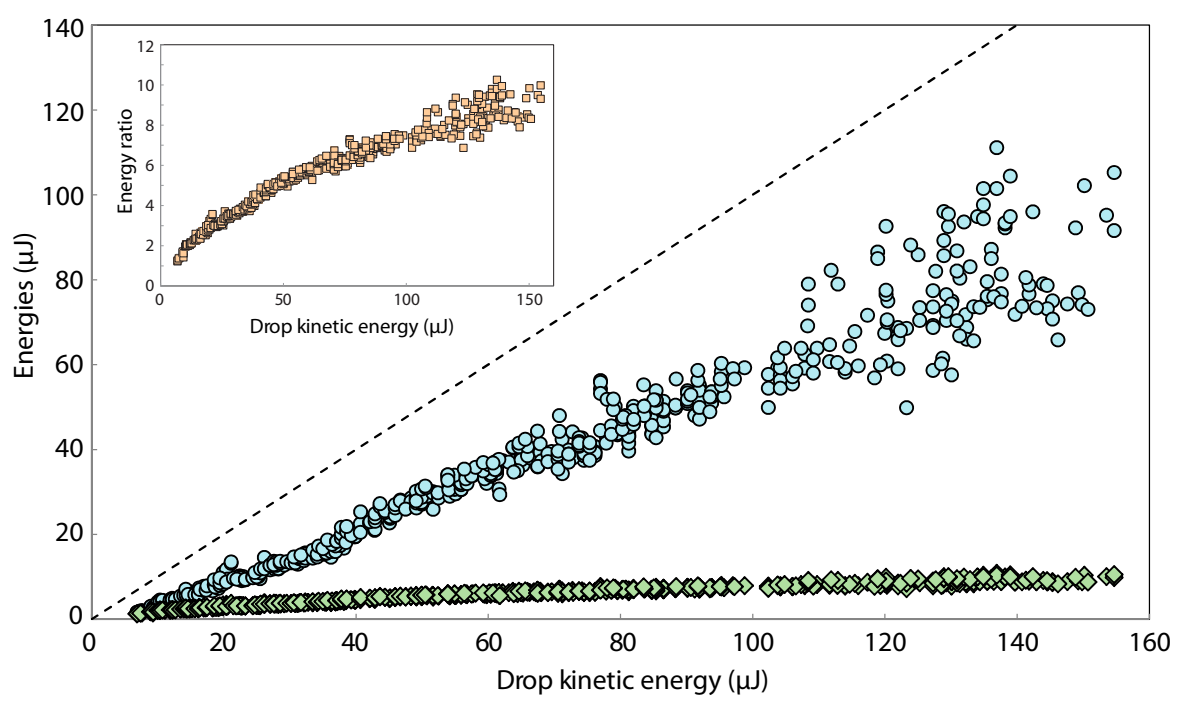

FIG. 2. Gravitational potential energy $U \sim \frac{\pi}{2} \rho_{l} g H^{2} R^{2}$ (blue circle), and capillary energy $C \sim 2 \pi \gamma H R$ (green diamond) of the cavity as a function of the falling drop kinetic energy $T=\frac{1}{2} M_{d} V_{i}^{2}$. The dashed line has a slope of 1 . In the insert the ratio between the gravitational and capillary energies, $U / C$, is also plotted as a function of the kinetic energy. This graph shows that for slow impacts, gravity and capillarity are comparable, and for high impact velocities, gravity clearly dominates the capillary effects.

form and the oscillations of the impacting drop [20,35,36]. However, since our work is related to the retraction dynamics and the jet and droplet characteristics, the precise shape of the impacting drop and the subsequent geometry of the cavity formed by this impact will not be considered in the present study. The kinetic energy of the remnant motion is also neglected.

Figure 2 displays the gravitational potential (blue circle) and capillary (green diamond) energy of the cavity as a function of the drop kinetic energy, while the insert of the figure presents the ratio between these two contributions. As expected, both energies increase as the impact velocity increases, indicating that the cavity size grows with the impact energy. Moreover, when kinetic energy is the lowest, it is converted equally in both potential and capillary energy, and as the impact kinetic energy is increased, the capillary energy increases only slightly and most of the drop kinetic energy is converted into the crater gravitational potential energy [37]. For the highest impact kinetic energies studied here, the gravitational energy is around ten times greater than capillary energy. Consequently, for most of the range of the drop impact velocity investigated, the energy stored by the cavity will be dominated by gravity; but, as we will see all along in this paper, the capillary waves cannot be neglected. Note that the sum of the two energy terms is not equal to the initial drop kinetic energy because the cavity-shape-dependent prefactors are only approximated and also because capillary waves and a small viscous dissipation consume energy. Previous studies have in fact shown that only a quarter of the impacting drop kinetic energy is converted to the potential energy of the cavity [21].

Figure 3 illustrates five typical drop impact events in water. In the five cases, the drop impacts the free surface of water, a cavity is shaped, and its relaxation gives rise to an upward vertical jet shooting out above the free surface. The cavity potential energy is then focused into a jet whose velocity and shape strongly vary from one case to another. Subsequently, the jet fragments [23], generating one or more droplets $[16,38]$. From the top sequence to the bottom one the impact velocity increases, leading to larger cavities and various jet dynamics and drop sizes. In the top sequence [Fig. 3(a)] the jet is quite small and slow. In the second and third sequences [Figs. 3(b) and 3(c)], the impact velocity is just a little higher but the jet dynamics is drastically different. In both cases the jet is very 
(a)

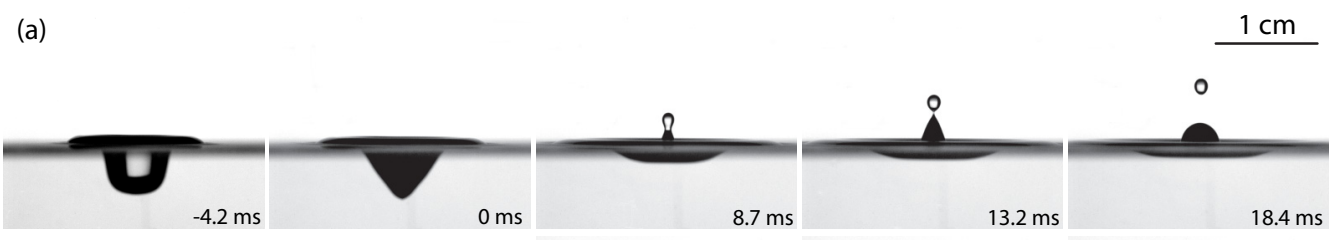

(b)

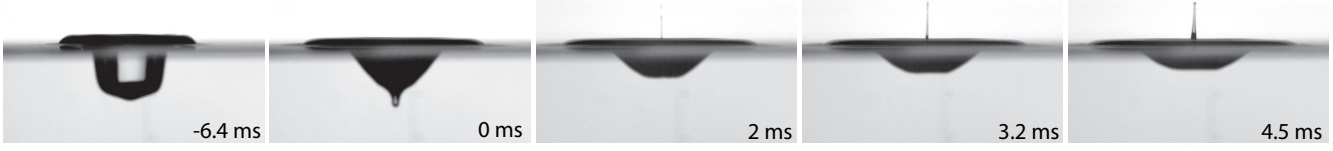

(c)

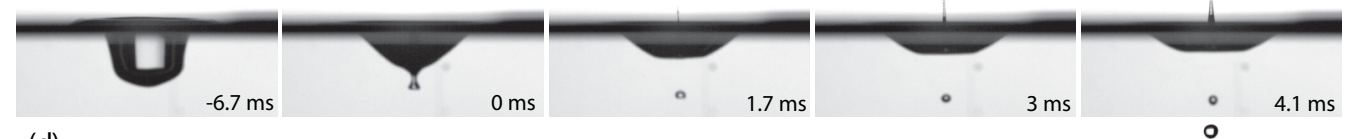

(d)

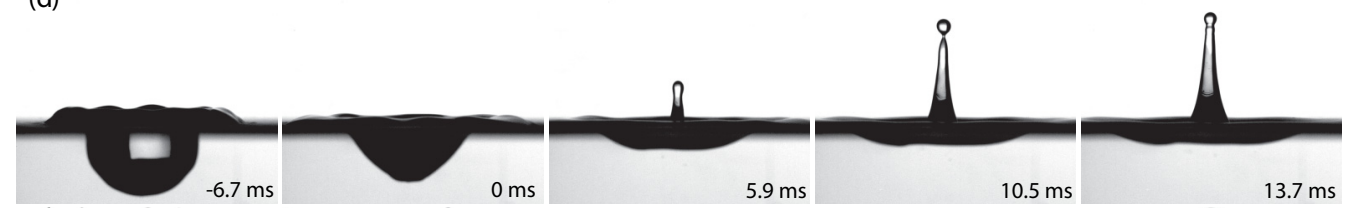

(e)
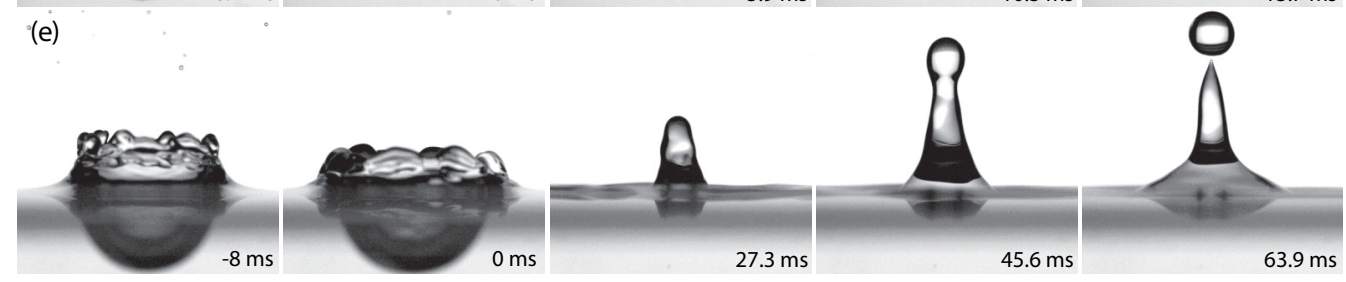

FIG. 3. Time sequences of a typical drop impact events at a free surface of water. In the five cases (a)-(e), the drop impacts the surface, a cavity is shaped, and the cavity relaxes giving rise to an upward vertical jet. The jet then fragments into droplets through successive end pinching events. From the top sequence to the bottom one, the impact velocity increases $(\mathrm{Fr}=93.5,127.5,145,348,926)$, leading to larger cavities and various jet dynamics and drop sizes. In each case, the time $t=0$ is taken right before the cavity reverses, and relative times are written on every other images. All the sequences have the same spatial scale, shown on the first sequence.

thin and fast and produces many very little droplets (not all of them on the pictures). The energy focusing process of the cavity relaxation is here very efficient. For the two last sequences, in Figs. 3(d) and 3(e), the drop impacts with even higher velocity and the jet dynamics revert to an expected behavior with a slower and fatter jet leading to large ejected droplets.

Between the first and the second columns, capillary waves, generated at the top of the cavity, propagate and focus at the bottom. This capillary retraction dynamics has been extensively described in the past $[21,39,40]$. These collapsing waves give rise to the vertical jet shooting out above the free surface as observed on the last columns. The second column presents the last image before the cavity reverses and a jet is formed; this defines our initial time $t=0$. We observe that the cavity is larger as drop velocity increases, in agreement with the energy increases of Fig. 2, and for the second and third sequences, where the jet is particularly thin and fast, the cavity exhibits a little subcavity. In some cases this little lump can detach a bubble [6,40], as observed on the sequence in Fig. 3(c). This bubble entrapment has been the topic of many studies, especially because of its application to 

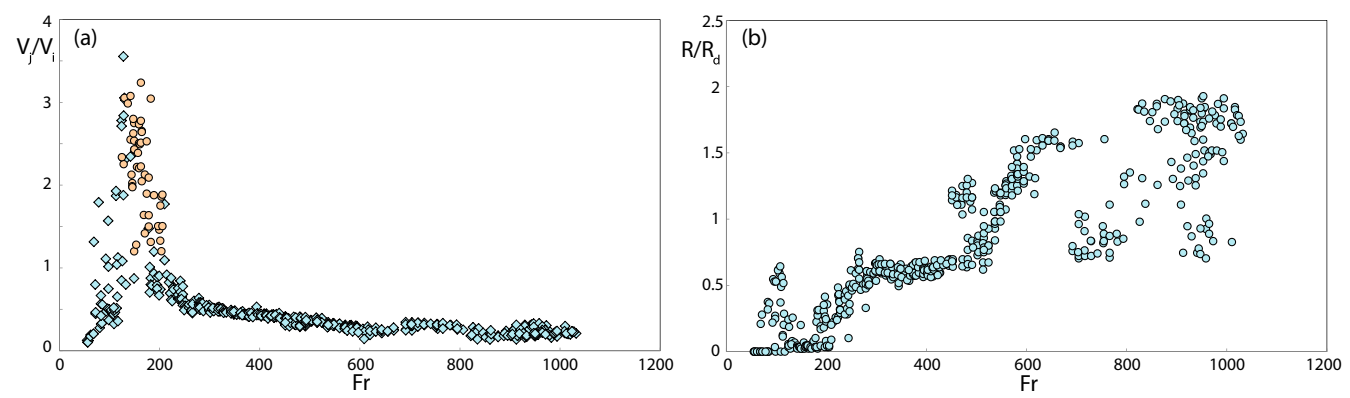

FIG. 4. (a) The jet velocity normalized by the drop impact velocity and (b) the top drop radius normalized by the impacting drop radius, following a drop impact on a free surface of water, as a function of the Froude number: $\mathrm{Fr}=V_{i}^{2} / g D_{d}$. The yellow circles indicate that the jet is accompanied by a bubble entrapment.

the study of the underwater noise of rain [33,37]. In these cases, it is known that the high-speed jet dynamics is induced by an efficient self-similar collapse of this subcavity [29,30,41].

\section{EJECTION VELOCITY AND DROP SIZE IN WATER}

To investigate more quantitatively these observations, Fig. 4(a) presents the jet velocity $\left(V_{j}\right)$ normalized by the drop impact velocity $\left(V_{i}\right)$ as a function of the impacting speed, quantified by the Froude number. The jet velocity is measured when the jet tip passes the free surface level. In some cases, the jet is already fragmented at this point and it is the top droplet velocity which is measured. When the drop of water is released below 80 millimeter height $(\mathrm{Fr} \lesssim 60)$ in a water tank, no jet is generated from the cavity collapse. By increasing the drop impact velocity ( $\mathrm{Fr} \gtrsim 60$ ), a jet starts to emerge with a low velocity. The jet velocity increases as the drop height is increased. This first regime ( $\mathrm{Fr} \lesssim 90)$ typically corresponds to the sequences in Fig. 3(a) with a slow and fat jet. The range of this regime is very small and it will not be discussed in the following.

Suddenly, for higher Froude number ( $\mathrm{Fr} \gtrsim 90)$, the jet velocity starts to take on much higher values together with a high variability, reaching velocities 3.5 to 4 times faster than the impacting drop. The cavity energy is here efficiently focused in the liquid jet. This high-speed jet regime has already been qualitatively observed $[21,22,42]$, and the very few quantitative results, mostly numerical $[40,43]$, provide values for the jet velocity in good agreement with our experiment. This regime corresponds to sequences in Figs. 3(b) and 3(c) where the jets are very thin. Such singular dynamics have also been observed for cavity collapses in slightly different contexts such as Faraday waves oscillations [41], bubble bursting [29,30], and drop impact on hydrophobic substrates [44]. In all these cases, the jet is induced by an efficient self-similar collapse of capillary waves focusing at the bottom of the cavity. This self-similar dynamics, driven by a balance between capillarity and inertia, can approach a finite time singularity, which is eventually regularized, focusing the energy in a very small subcavity and producing a fast and thin jet. In the present experiments, we expect such self-similar dynamics to occur, since a similar subcavity and subsequent fast and thin jet is observed in Figs. 3(b) and 3(c). This regime is called in the following the singular jet regime.

The yellow circles in Fig. 4(a) indicate that the focusing of the capillary waves entraps a bubble as observed in Fig. 3(c). Our results confirm the first observation of Liow [21] who showed in a phase diagram that this singular jet regime contains the bubble entrapment regime, defining the bubble entrapment regime as a subset of the high-speed jet regime. However, we notice that the entrapment of bubbles does not always accompany the formation of a high-speed jet [22], and that the velocity varies significantly in this regime. Indeed, bubble entrapment and jet velocity strongly depend on the way the capillary waves collapse, and small disturbances can have a strong influence on both the bubble and jet. Furthermore, since very small droplets are emitted in this regime, there are additional 
uncertainties related to the difficulty in identifying the first droplet. This singular regime is typically defined for Fr $\in[\sim 90, \sim 210]$, in good agreement with the literature [21,33].

For Froude numbers between $\sim 210$ and $\sim 1000$, the normalized jet velocity $V_{j} / V_{i}$ decreases very slowly. This regime will be discussed in more detail in Secs. VI and VII. We will show that jets in this regime are less influenced by the details of the capillary wave's collapse and result from the retraction of the whole cavity, this regime is thus called the cavity jet regime. In this case, we observe that cavity energy focusing is less and less efficient as the normalized velocity decreases slowly. Indeed, the increasing potential energy of the cavity with increasing impact velocity is mainly used to create bigger jets as it is observed in the last snapshot of sequences, Figs. 3(d) and 3(e). Similarly, more capillary waves are also radiated in all directions and the cavity becomes more and more wavy. Moreover, in this regime, bubble entrainment is suppressed. One reason might be that the wave front fails to reach the crater base before it reverses, inducing the formation of a thick and slow jet without bubble entrapment [42].

These different behaviors of the jet dynamics post impact have a deep influence on the detached droplet radius. Indeed, Fig. 4(b) presents the top jet droplet radius normalized by the impacting drop radius $\left(R_{d}\right)$ as a function of the Froude number. In the first regime, where jets are small and slow $(60 \lesssim \mathrm{Fr} \lesssim 90)$, the drop size increases. Suddenly, when the jet enters the singular jet regime, the drop becomes really tiny, below one tenth of the initial drop radius, with a minimum corresponding to the maximum jet velocity. This is in accordance with the jet shape observed in Figs. 3(b) and $3(c)$. For higher Froude numbers, Fr $\in[210,1000]$, the top drop size increases with Froude, despite a large variability. This confirms that jets become bigger with increasing impact velocity. Over the whole range, the top drop size is about the same size as the impacting drop $\left(\in\left[0.5 R_{d}, 2 R_{d}\right][26]\right)$ except in the singular jet regime.

At this point, it appears clearly that there is an excellent correlation between qualitative observation (Fig. 3) and quantitative measurement of jet velocity and top drop radius, in particular, between underwater bubble formation, high jet velocity, and numerous small droplets ejection. Moreover, this domain also matches the famous phase diagram of Pumphrey et al. [46] which presents the bubble entrapment regime. Our new observations, regarding drop size and velocity, add information to this phase diagram as it defines the bubble entrapment regime as a zone where the jets are faster and thinner, producing a very different kind of aerosol constituted of small, fast droplets.

\section{INFLUENCE OF LIQUID VISCOSITY}

We now investigate how the jet dynamics depends on the liquid viscosity. The jet eruption velocity $\mathrm{V}_{j}$, normalized by the drop impact velocity $\mathrm{V}_{i}$, is therefore plotted as a function of the Froude number in Fig. 5, for six different viscosities indicated on the graphs themselves. The first clear observation is that the jet dynamics depends strongly on viscosity in the singular regime, although the jet Reynolds number $\operatorname{Re}_{j}=\rho_{l} D_{d} V_{j} / \mu_{l}$ remains always much greater than 1 . Indeed, they get faster as the liquid viscosity increases from the viscosity of water (Fig. 4), up to $\mu_{l} \simeq 6 \mathrm{mPa} \mathrm{s}$ [Fig. 5(a)-5(c)]. This increase of the jet velocity with viscosity is surprising at first glance since usually viscosity brings dissipation and slows down processes. Here, this interesting increase of jet velocity with viscosity is a clear hallmark of the capillary effects. Indeed, viscous damping shelters the capillary wave's collapse from other perturbing ripples created at impact [29]. It thus allows the collapse to stay in its self-similar regime longer and to get closer to the finite time singularity $[41,45]$, generating a faster, a thinner jet.

We can also note that there is more reproducibility in this high-speed jet regime as viscosity increases. Indeed, from $6 \mathrm{mPa}$ s it seems that all the jets reach their maximum velocity and draw more precisely the envelop of this regime than for lower viscosities. This can be interpreted in the same way as the increase of jet velocity. By damping the remnant capillary ripples, viscosity allows the capillary dynamics to be more reproducible and less dependent on small disturbances.

For liquids more viscous than $\mu_{l}=6 \mathrm{mPa}$, the jet velocity in the singular jet regime decreases, up to $\mu_{l}=15 \mathrm{mPa}$ s where no more high-speed jets, nor bubble entrapment, are observed. Indeed, 

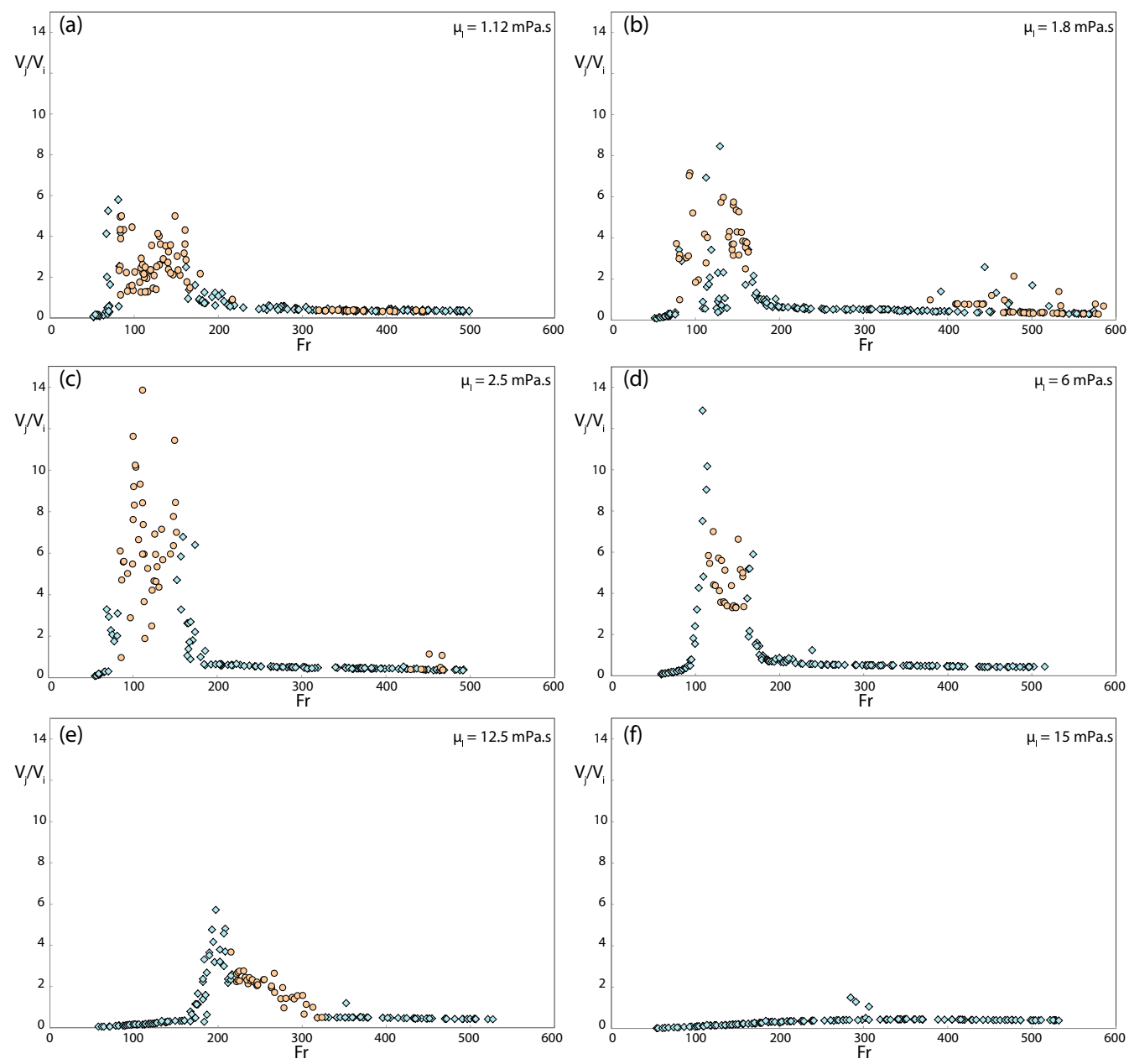

FIG. 5. Jet velocity $V_{J}$ normalized by the drop impact velocity $V_{i}$, following a drop impact on the free surface of viscous liquid with six different viscosities $\mu$, as a function of the Froude number: $\mathrm{Fr}=V_{i}^{2} / g D_{d}$. The yellow circles indicate that the jet is accompanied by a bubble entrapment.

at this viscosity almost no capillary waves can collapse efficiently enough to create bubbles and/or a singular jet. There are still jets but they are thick and slow, only induced by the cavity relaxation. Finally, we observe that the high Froude number regime, $\mathrm{Fr} \gtrsim 300$, is independent of liquid viscosity, showing its inviscid nature.

\section{CAPILLARY WAVE COLLAPSE AND CAVITY RETRACTION}

One of the most crucial influences of viscosity that we have not mentioned yet is the surprising appearance of a new underwater bubble regime at high Froude number $(\mathrm{Fr} \gtrsim 300)$ for viscosities up to $\mu_{l}=2.5 \mathrm{mPa}$ s, as indicated by the yellow circles on Figs. 5(a)-5(c). This second bubble regime is illustrated in Fig. 6(a). Because it appears at high Froude numbers, it corresponds to high drop impact velocity and thus strong corrugation of the free surface. We observe, indeed, that the base of the cavity is irregular in shape, perturbed by many random ripples, and the new bubble entrapment occurs from dimples in the cavity base. In our case, the liquid viscosity needs to be slightly above that of water $(1.1 \mathrm{mPa} \mathrm{s})$ to observe this regime. We need a hint of viscosity, probably because 


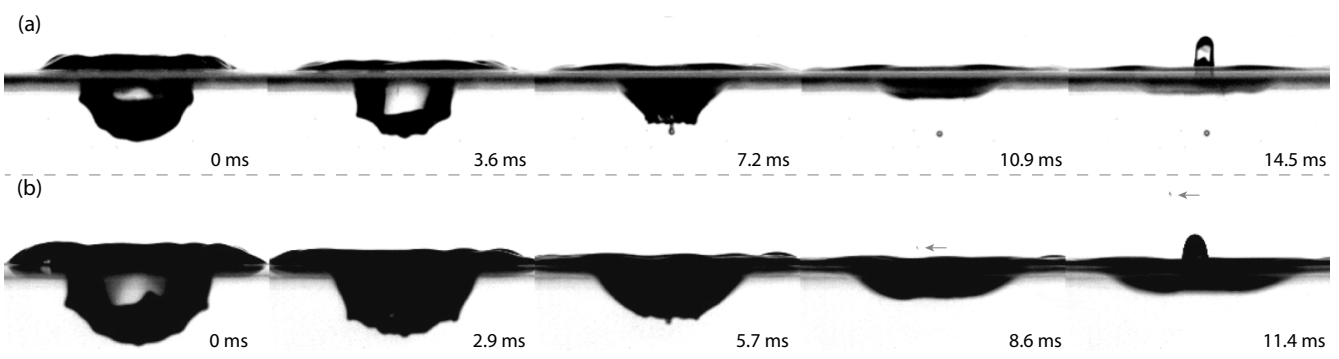

FIG. 6. (a) Sequence of a collapsing cavity following a drop impact on a slightly viscous liquid $\left(\mu_{l}=\right.$ $1.24 \mathrm{mPa} \mathrm{s}$ ) in the secondary bubble entrapment regime [high Froude number: Fr = 334.2, see Figs. 5(a)-5(c)]. In this case the bubble entrapment is followed by a slow, fat jet. (b) Sequence of a collapsing cavity following a drop impact on a slightly viscous liquid $\left(\mu_{l}=1.8 \mathrm{mPa}\right.$ s $)$ and high Froude number $(\mathrm{Fr}=573.8)$. The collapsing capillary waves eject a small droplet, pointed out by an arrow on the sequence. The droplet is followed by a slow, fat jet induced by the cavity collapse.

among all capillary waves generated at this high impact velocity, damping the smaller ones enables the bigger ones to propagate down to the cavity so as to collapse, form a subcavity, and eject a bubble. Note that this new bubble entrapment regime has been previously mentioned in water [21], but never illustrated nor discussed.

Finally, for $\mu_{l}=1.1 \mathrm{mPas}$ [Fig. 5(a)], we observe that this secondary bubble regime is not associated with any increase of the jet velocity. The capillary waves collapse, form a subcavity, and produce a bubble, but do not modify the thick, small, slow jet's behavior. This is surprising and brings out the question of the role of the capillary wave in the jet dynamics in this regime.

By increasing the viscosity a little more (up to $\mu_{l}=1.8 \mathrm{mPas}$ ), this secondary bubble regime allows sometimes the ejection of a faster "jet" which superposes on the thick and slow jet regime. A typical sequence of this behavior is illustrated in Fig. 6(b). We observe that the faster "jet" is actually just a single tiny droplet, pointed out by an arrow on the sequence. This droplet ejected by the capillary wave collapse is then followed by a fat and slow jet, comparable to the one in Figs. 3(e) and 6(a), induced by the whole cavity relaxation. Therefore, in this regime, the self-similar collapse of a capillary subcavity can enable the ejection of a small droplet but cannot modify the thick jet dynamics, at variance with the singular jet regime.

In fact, two different mechanisms are at play here: the capillary wave collapse and the cavity retraction. In the singular jet regime, the capillary wave collapse was violent enough to trigger the cavity retraction and to eject a single thin, fast jet. The capillary collapse and the cavity relaxation were therefore correlated. In the cavity jet regime presented here, the cavity is too big and its retraction dynamics seems independent of the collapse of some capillary waves traveling on the cavity surface. This leads here to two decorrelated events: a fast and small drop is sometimes ejected by an efficient capillary wave collapse while a slow and fat jet is produced later by cavity relaxation. A random capillary collapse can then not produce a jet if it is not fed by the cavity retraction. In this case the scale of the jet is therefore totally different than the scale of the drop, which are two different objects.

In conclusion, this experiment enables us to distinguish between cavity retraction and the singular collapse of capillary waves. When the capillary and cavity scales become too different they cannot act together anymore to create the same object. This defines $\mathrm{Fr}=300$ as the limit for having a real singular jet. Note that for much larger cavities such as those obtained by large interface deformation [31], capillary wave collapse and cavity retraction are obviously expected to be decorrelated as well.

\section{GRAVITATIONAL OR CAPILLARY CAVITY RETRACTION}

At this point we are convinced that the high-speed jets in the singular jet regime or the ejected droplets in the cavity jet regime have a dynamics dominated by capillarity because they are the direct 

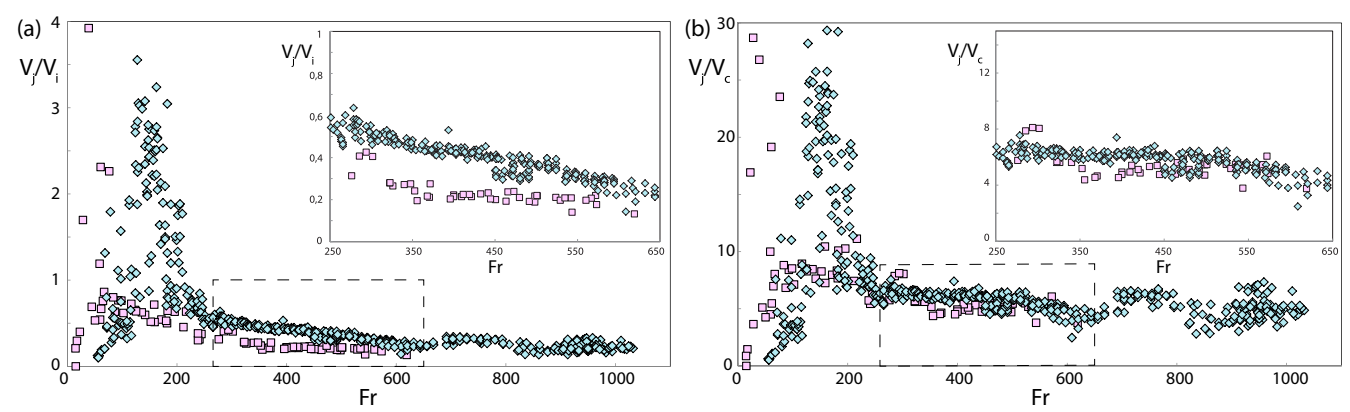

FIG. 7. Jet velocity $V_{J}$ normalized by (a) the drop impact velocity $V_{i}$ as a function of the Froude number, Fr $=V_{i}^{2} / g D_{d}$, and by (b) the capillary velocity $V_{c}=\sqrt{\gamma /\left(\rho_{l} D_{d}\right)}$ as a function of the Froude number. The two curves correspond to a drop impacting the free surface of water (turquoise diamond) and ethanol (pink square) with respective surface tensions $\gamma=0.072$ and $0.022 \mathrm{mN} \mathrm{m}^{-1}$. Note that the drop diameter $D_{d}$ is the same in water and in ethanol. The insets zoom in on the zones of interest and exhibit a collapse of the two curves in (b), with a plateau regime at high Froude numbers, showing that $V_{j} \propto V_{c}$.

consequence of a capillary wave collapse. Here, we wish to discuss the force inducing the cavity retraction in the cavity jet regime where capillary wave collapse cannot entrain the cavity reversal. In this high Froude number regime we have observed that most of the cavity energy is due to the gravity contribution (Fig. 2). It is thus tempting to question whether the collapse of this gravitational cavity is also gravity dominated, as observed for a much bigger cavity [31], or is still dominated by surface tension. We have thus performed experiments with a liquid which has a different surface tension than water, namely ethanol whose surface tension is $\gamma=0.022 \mathrm{mN} \mathrm{m}^{-1}$. Figure 7(a) draws the velocity ratio $V_{j} / V_{i}$ both for water (turquoise diamond) and ethanol (pink square) as function of the Froude number, showing clearly a dependence of the liquid jet with the surface tension for Froude numbers larger than 200, although the profiles of the two curves are similar.

Surface tension therefore clearly plays a role in the relaxation process that we need to disentangle from the influence of gravity. To do this, we plot the same jet velocities as functions of the Froude number both for ethanol and water on Fig. 7(b), but rescaled now by the capillary velocity, defined for each liquid by

$$
V_{c}=\sqrt{\frac{\gamma}{\rho_{l} D_{d}}} .
$$

We observe a clear collapse of the two curves (see the insets for a zoom in the zone of interest), which, in addition, exhibit a plateau regime in this high Froude regime showing that $V_{j} \propto V_{c}$.

In conclusion, for these high Froude numbers (Fr 300-1000), although the cavity is gravity dominated, its retraction and the resulting jet dynamics are selected by capillary forces. We have also already pointed out that in this regime, the jet velocity is almost independent of the liquid viscosity. In other words, we have demonstrated that the Weber number built on the jet velocity $\mathrm{We}_{j}=\rho_{l} V_{j}^{2} D_{d} / \gamma$ is constant here and, in particular, is independent of the impact velocity, gravity, and viscosity. As a consequence, this shows that the variation of velocity with cavity depth is different from previous studies. Indeed, with increasing cavity depth, jet velocity decreases for submillimeter $\operatorname{cavities}\left(V_{j} \propto H^{-1 / 3}\right)$ [29], and increases for centimeter cavities $\left(V_{j} \propto H^{3 / 2}\right)$ [31]; and here, for intermediate cavity sizes, the jet velocity is constant $\left(V_{j} \propto H^{0}\right)$.

\section{CONCLUSION}

In this paper, we have characterized experimentally the jets and the resulting ejected droplets that are formed by the collapse of the cavity created by the impact of a drop on a pool of the same aqueous liquid. We have shown that very different jets can spring (fast, thin, slow, fat, ...), depending on the 
impact parameters, characterized here by the Froude number. After presenting and discussing the jet velocity and drop radius variation as a function of the impact Froude number, we examine the influence of the liquid viscosity and surface tension on the jet velocity. Thus, we are now able to disentangle the different mechanisms at play in the cavity retraction and subsequent jet dynamics and define two distinct regimes: the singular jet and the cavity jet regimes.

In the singular jet regime, the jet is induced by the self-similar collapse of the focusing capillary waves at the bottom of the retracting cavity. This type of collapse is known to tend toward a finite time singularity and to be eventually regularized at the scale of the emitted jet. Such regularization can have different origins, from viscous dissipation at short scale to interface fluctuations, for instance. In addition, in this regime, this intense wave collapse is able to entrain the cavity reversal and therefore gives birth to a coherent high-speed vertical jet, thin, detaching very small droplets, and exhibiting very variable velocities. This self-similar collapse is also able to entrap underwater bubbles. This regime strongly depends on the liquid viscosity as the focusing capillary waves do. In particular, the jet velocity first increases with viscosity, then decreases before this regime disappears at high viscosity.

The cavity jet regime is defined for higher Froude numbers. Here, the jets are fat and slow. The first surprise is that for these high impact velocities, the jet velocity is totally capillary selected. Indeed, the mechanism which brings this crater back to a flat equilibrium is complex [20] and our results show that surface tension is crucial there although the stored energy in the cavity is mostly due to gravity. Consequently, the jet velocity is simply proportional to the capillary velocity $\sqrt{\gamma /\left(\rho_{l} D_{d}\right)}$ and is in particular independent of viscosity and impact velocity. In this regime, capillary waves obviously keep focusing and collapsing at the bottom of the cavity, but their collapses are here decorrelated from the whole cavity retraction. This is probably due to a large gap in the characteristic scales between the waves and the cavity. Therefore, in some cases the capillary wave collapse can entrap a bubble and/or eject a drop. But this behavior, which is the hallmark of a self-similar collapse usually entraining a high-speed jet, does not affect the retraction of the whole cavity in this regime and in particular never triggers any thin and fast jet. This experiment therefore enables us to correlate, in the singular regime, and decorrelate, in the cavity jet regime, the capillary wave collapse and the cavity retraction.

Finally, our results reveal that the sizes and velocities of the droplets ejected by the central jet after impact of a drop on a pool are far from following a simple monotonic relation with the impact parameters. This aerosol generation should be investigated in great detail in the future depending on the physical application (sea sprays or petrichor, for instance) [47].

[1] A. M. Worthington and R. S. Cole, Impact with a liquid surface studied by the aid of instantaneous photography. Paper II, Philos. Trans. R. Soc. London A 194, 175 (1900).

[2] H. Edgerton and J. Killian, Flash! Seeing the Unseen by Ultra High-speed Photograph (Branford, Boston, 1954).

[3] H. Edgerton, E. Jussim, and G. Kayafas, Stopping Time: The Photographs of Harold Edgerton (Abrams, New York, 1987).

[4] A. v. d. Bos, M.-J. v. d. Meulen, T. Driessen, M. v. d. Berg, H. Reinten, H. Wijshoff, M. Versluis, and D. Lohse. Velocity Profile Inside Piezoacoustic Inkjet Droplets in Flight: Comparison Between Experiment and Numerical Simulation, Phys. Rev. Appl. 1, 014004 (2014).

[5] A. Antkowiak, B. Audoly, C. Josserand, S. Neukirch, and M. Rivetti, Instant fabrication and selection of folded structures using drop impact, Proc. Natl. Acad. Sci. USA 108, 10400 (2011).

[6] A. Prosperetti, L. A. Crum, and H. C. Pumphrey, The underwater noise of rain, J. Geophys. Res. 94, 3255 (1989).

[7] R. Guigon, J.-J. Chaillout, T. Jager, and G. Despesse, Harvesting raindrop energy: Experimental study, Smart Materials and Structures 17, 015039 (2008). 


\section{MICHON, JOSSERAND, AND SÉON}

[8] N. Laan, K. G. de Bruin, D. Bartolo, C. Josserand, and D. Bonn, Maximum Diameter of Impacting Liquid Droplets, Phys. Rev. Appl. 2, 044018 (2014).

[9] H. J. Melosh and B. A. Ivanov, Impact crater collapse, Annu. Rev. Earth Planet. Sci. 27, 385 (1999).

[10] Y. S. Joung and C. R. Buie, Aerosol generation by raindrop impact on soil, Nat. Commun. 6, 01 (2015).

[11] A. L. Yarin, Drop impact dynamics: Splashing, spreading, receding, bouncing..., Annu. Rev. Fluid Mech. 38, 159 (2006).

[12] C. Josserand and S. Thoroddsen, Drop impact on a solid surface, Annu. Rev. Fluid Mech. 48, 365 (2016).

[13] P. V. Hobbs and A. J. Kezweeny, Splashing of a water drop, Science 155, 1112 (1967).

[14] T. Gilet and L. Bourouiba, Fluid fragmentation shapes rain-induced foliar disease transmission, J. R. Soc. Interface 12, 20141092 (2015).

[15] E. Villermaux and B. Bossa, Drop fragmentation on impact, J. Fluid Mech. 668, 412 (2011).

[16] D. C. Blanchard, The electrification of the atmosphere by particles from bubbles in the sea, Prog. Oceanogr. 1, 73 (1963).

[17] M. Rein, Phenomena of liquid drop impact on solid and liquid surfaces, Fluid Dyn. Res. 12, 61 (1993).

[18] B. Peck and L. Sigurdson, The three-dimensional vortex structure of an impacting water drop, Phys. Fluids 6, 564 (1994).

[19] P. Shankar and M. Kumar, Vortex rings generated by drops just coalescing with a pool, Phys. Fluids 7, 737 (1995).

[20] M.-J. Thoraval, L. Yangfan, and S. T. Thoroddsen, Vortex-ring-induced large bubble entrainment during drop impact, Phys. Rev. E 93, 033128 (2016).

[21] L. J. Liow, Splash formation by spherical drops, J. Fluid Mech. 427, 73 (2001).

[22] M. Rein, The transitional regime between coalescing and splashing drops, J. Fluid Mech. 306, 145 (1996).

[23] E. Ghabache and T. Séon, Size of the top jet drop produced by bubble bursting, Phys. Rev. Fluids 1, 051901 (2016).

[24] J. M. Gordillo and S. Gekle, Generation and breakup of Worthington jets after cavity collapse. Part 2. Tip breakup of stretched jets, J. Fluid Mech. 663, 331 (2010).

[25] J. Eggers and E. Villermaux, Physics of liquid jets, Rep. Prog. Phys. 71, 036601 (2008).

[26] E. Castillo-Orozco, A. Davanlou, P. K. Choudhury, and R. Kumar, Droplet impact on deep liquid pools: Rayleigh jet to formation of secondary droplets, Phys. Rev. E 92, 053022 (2015).

[27] O. G. Engel, Crater depth in fluid impacts, J. Appl. Phys. 37, 1798 (1966).

[28] H. Medwin, J. A. Nystuen, P. W. Jacobus, L. H. Ostwald, and D. E. Snyder, The anatomy of underwater rain noise, J. Acoust. Soc. Am. 92, 1613 (1992).

[29] E. Ghabache, A. Antkowiak, C. Josserand, and T. Séon, On the physics of fizziness: How bubble bursting controls droplets ejection, Phys. Fluids 26, 121701 (2014).

[30] L. Duchemin, S. Popinet, C. Josserand, and S. Zaleski, Jet formation in bubbles bursting at a free surface, Phys. Fluids 14, 3000 (2002).

[31] E. Ghabache, T. Séon, and A. Antkowiak, Liquid jet eruption from hollow relaxation, J. Fluid Mech. 761, 206 (2014).

[32] A. Benusiglio, D. Quéré, and C. Clanet, Explosions at the water surface, J. Fluid Mech. 752, 123 (2014).

[33] H. N. Oguz and A. Prosperetti, Bubble entrainment by the impact of drops on liquid surfaces, J. Fluid Mech. 219, 143 (1990).

[34] A. Bisighini, G. E. Cossali, C. Tropea, and I. V. Roisman, Crater evolution after the impact of a drop onto a semi-infinite liquid target, Phys. Rev. E 82, 036319 (2010).

[35] S. T. Thoroddsen, T. G. Etoh, K. Takehara, N. Ootsuka, and Y. Hatsuki, The air bubble entrapped under a drop impacting on a solid surface, J. Fluid Mech. 545, 203 (2005).

[36] A.-B. Wang, C.-C. Kuan, and P.-H. Tsai, Do we understand the bubble formation by a single drop impacting upon liquid surface? Phys. Fluids 25, 101702 (2013).

[37] A. Prosperetti and H. N. Oguz, The impact of drops on liquid surfaces and the underwater noise of rain, Annu. Rev. Fluid Mech. 25, 577 (1993).

[38] D. E. Spiel. The number and size of jet drops produced by air bubbles bursting on a fresh water surface, J. Geophys. Res. 99, 10289 (1994). 
[39] A. H. Woodcock, C. F. Kientzler, A. B. Arons, and D. C. Blanchard. Giant condensation nuclei from bursting bubbles, Nature 172, 1144, (1953).

[40] B. Ray, G. Biswas, and A. Sharma, Regimes during liquid drop impact on a liquid pool, J. Fluid Mech. 768, $492(2015)$.

[41] B. W. Zeff, B. Kleber, J. Fineberg, and D. P. Lathrop, Singularity dynamics in curvature collapse and jet eruption on a fluid surface, Nature 403, 401 (2000).

[42] D. Morton, M. Rudman, and L. Jong-Leng, An investigation of the flow regimes resulting from splashing drops, Phys. Fluids 12, 747 (2000).

[43] B. Ray, G. Biswas, and A. Sharma, Bubble pinch-off and scaling during liquid drop impact on liquid pool, Phys. Fluids 24, 082108 (2012).

[44] D. Bartolo, C. Josserand, and D. Bonn, Singular Jets and Bubbles in Drop Impact, Phys. Rev. Lett. 96, 124501 (2006).

[45] M. P. Brenner, Fluid mechanics: Jets from a singular surface, Nature 403, 377 (2000).

[46] H. C. Pumphrey and P. A. Elmore, The entrainment of bubbles by drop impacts, J. Fluid Mech. 220, 539 (1990).

[47] E. Ghabache, G. Liger-Belair, A. Antkowiak, and T. Séon, Evaporation of droplets in a champagne wine aerosol, Sci. Rep. 6, 25148 (2016). 